\title{
Frequency maps as a probe of secular evolution in the Milky Way
}

\author{
Monica Valluri \\ Department of Astronomy, University of Michigan, Ann Arbor, MI 48104, USA \\ email: mvalluri@umich.edu
}

\begin{abstract}
The frequency analysis of the orbits of halo stars and dark matter particles from a cosmological hydrodynamical simulation of a disk galaxy from the MUGS collaboration (Stinson et al. 2010) shows that even if the shape of the dark matter halo is nearly oblate, only about $50 \%$ of its orbits are on short-axis tubes, confirming a previous result: under baryonic condensation all orbit families can deform their shapes without changing orbital type (Valluri et al. 2010). Orbits of dark matter particles and halo stars are very similar reflecting their common accretion origin and the influence of baryons. Frequency maps provide a compact representation of the 6-D phase space distribution that also reveals the history of the halo (Valluri et al. 2012). The 6-D phase space coordinates for a large population of halo stars in the Milky Way that will be obtained from future surveys can be used to reconstruct the phase-space distribution function of the stellar halo. The similarity between the frequency maps of halo stars and dark matter particles (Fig. 1) implies that reconstruction of the stellar halo distribution function can reveal the phase space distribution of the unseen dark matter particles and provide evidence for secular evolution. MV is supported by NSF grant AST-0908346 and the Elizabeth Crosby grant.
\end{abstract}

Keywords. methods: n-body simulations, Galaxy: evolution, galaxies: halos, galaxies: dark matter
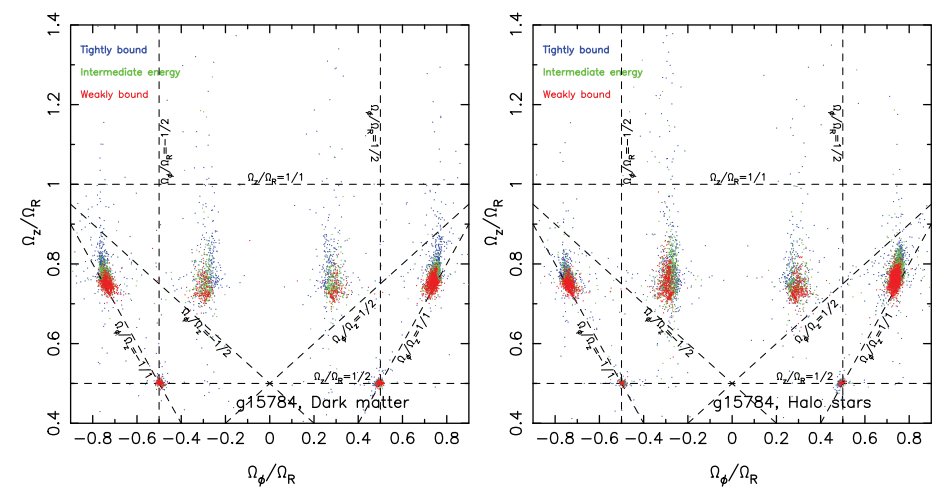

Figure 1. Frequency maps in cylindrical coordinates for $\sim 10^{4}$ halo orbits in a cosmological disk galaxy. Color represents total energy of each particle. The resonances at $\Omega_{z} / \Omega_{R}=\left|\Omega_{\phi} / \Omega_{R}\right|=0.5$ correspond to a "thin shell" family: its prominence even in this hierarchical simulation is evidence of secular resonant trapping of halo particles by the growth of the stellar disk.

\section{References}

Stinson, G. S., et al. T. 2010, MNRAS, 408, 812

Valluri, M., Debattista, V. P., Quinn, T., \& Moore, B. 2010, MNRAS, 403, 525

Valluri, M., Debattista, V. P., Quinn, T. R., Roškar, R., \& Wadsley, J. 2012, MNRAS, 419, 1951 